\title{
Soft-Error-Rate Adaptive Intervals for Low-Overhead Checkpoint Mechanism
}

\author{
Wentao Jia*, Chunyuan Zhang \\ National Laboratory for Parallel and Distributed Processing, National University of Defense Technology, \\ Changsha, China. \\ * Corresponding author. Tel.: +8613739079499; email: wtjia@nudt.edu.cn \\ Manuscript submitted September 4, 2015; accepted December 8, 2015. \\ doi: 10.17706/ijcee.2015.7.6.387-398
}

\begin{abstract}
Soft errors are increasingly important threats to the reliability of integrated circuits. Chips manufactured in advanced technologies show variations in SER caused by variations in the process parameters. Ongoing reduction of feature sizes and complexity of operating environment (temperature, voltage, radiation pressure and so on), SER variation is increasingly manifesting. Checkpoint is one of the most popular recovery method used for many systems, and the intervals of checkpoint can obviously influence performance. However, optimal intervals of checkpoint rely on SER. Theoretically speaking, $S E R$ adaptive checkpoint (SACP) which dynamically match checkpoint intervals with real time SER can improve checkpoint overhead under variable SER. But benefits of SACP are relative with SER variation. We give a mathematical model of $S E R$ variation and proposal a way to predict $S E R$ based errors occurred most currently. Results show high accuracy of SER prediction and much overhead improvement of SACP.
\end{abstract}

Key words: Soft-error-rate, adaptive, error variation, checkpoint intervals.

\section{Introduction}

Soft errors including SBU (single-bit-upset) and MBU (multiple-bit upset) are increasingly important threats to the reliability of integrated circuits fabricated in advanced CMOS technologies. Researchers expect an aggregate effect on soft-error rate (SER) of a chip. The error rate at 16-nm may be almost 100 times that at $180 \mathrm{~nm}$ [1]. Ongoing reduction of feature sizes has increased the probability that a single particle causes an MCU (Multiple-cell upset). It has been observed that a single neutron caused more than 50 bit flips in an SRAM of 65nm [2]. During neutron-accelerated SER tests of SRAMs in advanced processes, it is not unusual to observe that more than $50 \%$ of the upset events are MCUs [2], implying ECC techniques generally applied are more and more not capable of protecting systems.

Chips manufactured in advanced technologies gradually show variability on SER caused by variation in the process parameters. Voltage supply variability, voltage threshold variability and channel length variability at the lower level are directly associated with overall circuits at the higher level, not only performance variability and power variability, but also SER variability. Process variability causes that the $S E R$ vulnerability of an SRAM bit cell is not the same for its two data states. Experimental results for a 90-nm embedded SRAM showed that the differences can be almost a factor of 4 [3]. SRAM bit cells are symmetric by design, however sequential logic are not. As a result, the $S E R$ of sequential logic usually varies with the data state (0/1) and clock state (HIGH/LOW), the differences can be nearly 10X [4]. SER differences caused by process variability while chips manufactured and designed will eventually show $S E R$ 
variations while systems running, meaning SER of systems may not keep constant but change from time to time.

Moreover, SER is highly associated with operating environment (temperature, voltage, radiation pressure and so on), which is not constant always but variable sometime. As computing is emerging anywhere and any-time, operating environment of many systems are variable, such as spacecraft, smart city system, unmanned verticraft, intelligent vehicle. As cars drive from New York City to Denver, CO, USA, SER increase 3.5X (due to altitude increased by 1.6KM) [5]. Many systems employ dynamic mechanisms to decrease power or energy, like DVFS, which also making voltage or frequency variable. Ongoing reduction of feature sizes and complexity of operating environment, SER variation is increasingly manifesting.

One of the most popular recovery methods is checkpoint. During checkpointing shown in Fig. 1, application entire statement is written to storage so that in occurrence of errors application can resume its work from the last checkpoint rather than from the beginning. $T_{\text {ex }}$ in Fig. 1 is execution time of application, $T_{\mathrm{ov}}$ is time to store application statement, $T_{r}$ is time to recovery application, $T_{\text {solve }}$ is solve time for application which is equal to $N \times T_{\text {ex }}, N$ is the number of passed segments required to complete a calculation. Intervals of checkpoint $T_{\text {ex }}$ can obviously influence checkpoint performance.

Based on checkpoint model proposed by Daly [6], Fig. 2 shows how the performance overhead varies with checkpoint intervals, overhead $=\left(T_{\text {total }}-T_{\text {solve }}\right) / T_{\text {solve. }}$ Optimal checkpoint interval for MTTE (mean time to errors) $=100 \mathrm{~min}$ is $21 \mathrm{~min}$. Keeping the interval while $S E R$ changing to MTTE $=10 \mathrm{~min}$ or MTTE $=1000 \mathrm{~min}$, overhead will be $550 \%$ or $12 \%$. However, overhead at optimal interval is $235 \%$ or $7 \%$. So SER adaptive intervals of checkpoint can reduce overhead for variable SER. Theoretically speaking, SER adaptive checkpoint (SACP) which dynamically matches checkpoint intervals with real time $S E R$ can improve checkpoint overhead under variable $S E R$.

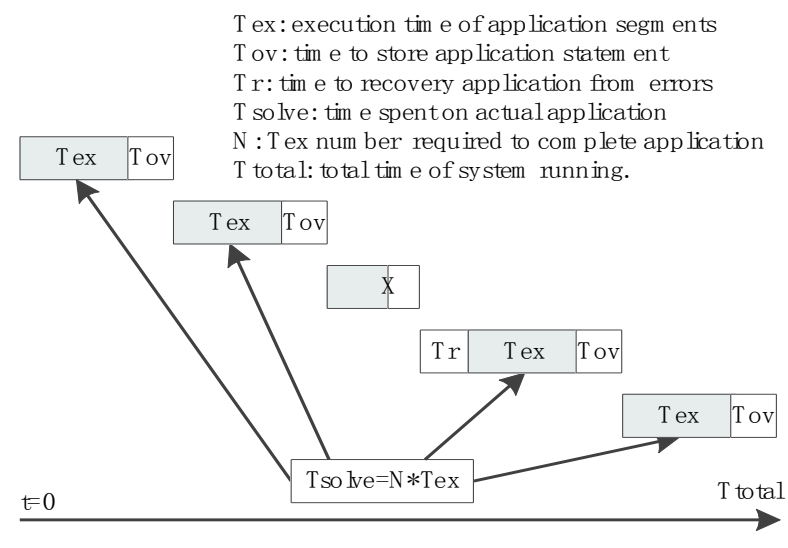

Fig. 1. checkpoint model.

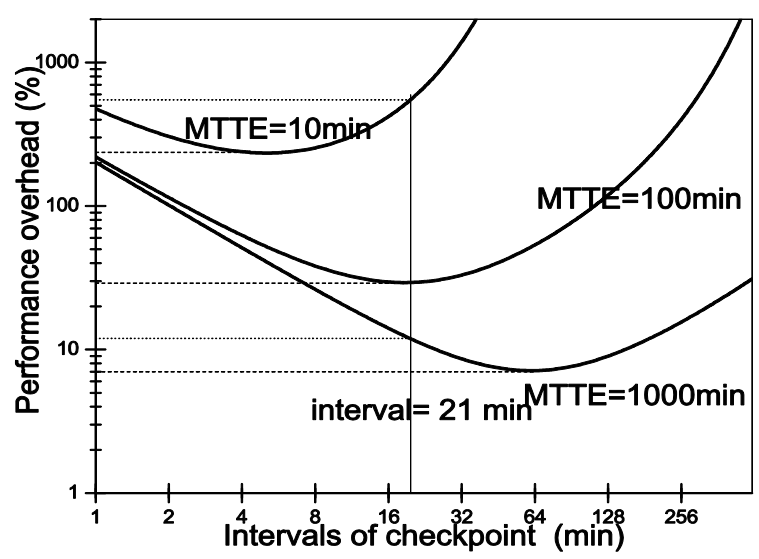

Fig. 2. Checkpoint overhead.

There are two points to reduce overhead for present checkpoint mechanism: reducing MTTE or time overhead to update checkpoint. Differently, the view of SER adaptive checkpoint (SACP) is that analyze occurrence of errors more carefully and match checkpoint interval with real time $S E R$ dynamically. Benefit of SACP is relative with SER variation (how large the variation takes and how long it keeps), so we firstly have to evaluate impact of $S E R$ variability on $S E R$ adaptive checkpoint.

We make the following contribution in this paper: 1 . Based on how SER affected by temperature, voltage, radiation pressure and so on, we give a mathematical model with four parameters to quantized $S E R$ variation. 2. We study impact of variable SER on overhead of present checkpoint and SER adaptive checkpoint, indicating optimal benefits of SACP. 3. We proposal a way to predict SER based dynamic prediction window, showing practical benefits of SACP. 


\section{Background and Related Works}

This paper focus on decreasing checkpoint overhead under variable SER. SER can be described by mean time to errors(MTTE) or number of errors occurred in unit time, and this paper will use later, that is $S E R=1 /$ MTTE.

SER variation. Soft errors are caused by either neutrons generated by cosmic radiation interacting with the earth's atmosphere or alpha particles emitted by radioactive impurities that are present in chips and package materials. SER can be get from the following equation [7]:

$$
S E R=\text { Constant } \times \text { Flux } \times \text { Area } \times e^{-Q \text { crit } / Q \text { coll }}
$$

Flux is the alpha or neutron flux experienced by the circuit, Area is the effective diffusion area, Qcrit is Critical charge, and $Q$ coll is the collection efficiency. Flux varies with altitude, location on the earth, concrete thickness of building, solar activity and so on. $Q$ crit depends on the supply voltage and temperature. Table 1 lists variable factors related to $S E R$.

Table 1. Variable Factors Related to SER

\begin{tabular}{l|l|l}
\hline variable factor $X$ & how $S E R$ varies with $X$ & amplitude of $S E R$ variation* \\
\hline Altitude & SER0 $\times \mathrm{e}^{0.9 x-0.03 x^{\times} \times}[5]$ & over 100 (sea level to flight level $12 \mathrm{KM})$ \\
\hline Latitude & & $3(20 \mathrm{~N}-60 \mathrm{~N})$ \\
\hline Concrete Thickness & SER0 $\times \mathrm{e}^{-2.7 x}[8]$ & $15(0-1 \mathrm{~m})$ \\
\hline Solar Cycle & & $1.3[8]($ day to next day) \\
& & $1.6[8]($ month to next month) \\
\hline Voltage & $S E R 0 \times \mathrm{e}^{-1.3 x+0.3}[9]$ & $3(0.4 \mathrm{~V} \sim 1.0 \mathrm{~V}) @ 45 \mathrm{~nm}$ \\
\hline Temperature & $\approx \exp [10]$ & $3(25 \mathrm{C}-110 \mathrm{C})$ \\
\hline
\end{tabular}

Form Table 1, we can see SER exponentially varies with most variable factor, for variable factor $X \in$ \{Altitude, Thickness, Voltage, Temperature\}, SER could be

$$
\operatorname{SER}(X)=S E R 0 \times e^{C X^{*}(X-X 0)}
$$

$X 0$ and $S E R 0$ are a pair known value of $X$ and corresponding $S E R, C X$ is a constant indicating amplitude of $S E R$ variation with $X$ variation per unit. Take altitude for example, $C X=0.8$, meaning $S E R$ will increase $e^{0.8}$ per kilo-meter higher. If $C X<0$, indicating a decrease of $S E R$ while $X$ increase, such as voltage and thickness.

In summary, amplitude of $S E R$ variation caused by operating environment can be from several to a hundred and caused by process variability can be ten. Considering both, amplitude of SER variation can be nearly thousand.

Checkpoint. Checkpoint technique is commonly used to recover from application failure. One aspect of employing checkpoint is properly assigning checkpoint intervals $T_{\text {ex. }}$ Daly[6] proposal a accurate ways based $S E R$ and $T_{\text {ov }}$ to determine the optimum checkpoint interval. We also use Daly's method to determine checkpoint intervals in this paper.

Adaptive checkpoint. Previous research improving overhead focused primarily on decreasing the checkpoint time of data transferred [11], [12], while relying on constant checkpoint frequency. Gerofi [13] proposal an algorithm that adapts dynamically to the properties of the workload being executed, such as changes in the number of dirtied memory pages, network and disk I/O operations, as well as to the network bandwidth available for replication. The results show benefits of adaptive checkpoint. The most difference with previous works is that we are focusing on SER variation which has rarely been studied. 


\section{Modeling SER Variation}

We define $S E R$ at time $t$ as $S E R(t)$, let initial $S E R$ be $S E R 0$, so constant $\operatorname{SER}$ is $\operatorname{SERc}(t)=\operatorname{SER}(0)=\operatorname{SER} 0$. Variable $S E R$ according to Eq. (1) is

$$
\operatorname{SERv}(t)=\operatorname{SER} 0 \times e^{C x^{\times}(X(t)-X 0)}
$$

$X(t)$ usually relevant to system type, application field, operating environment and so on. Variation of $X(t)$ based $X 0$ is shown as Eq.(3)

$$
X(t)=\left\{\begin{array}{lr}
X 0 & t \% T \leq T-T_{v} \\
X 0+a \times \sin \left(\pi\left(t-T+T_{v}\right) / T_{v}\right) & t \% T>T-T_{v}
\end{array}\right.
$$

With $a$ indicating the largest amplitude increased or decreased, $T$ is cycle time of variation and we use the mod "\%" operator to make it, $T_{v}$ is time for variation amplitude $a$ (shown in Eq.(3)) sustained. Here we use $\sin ()$ function to describe variation for a complicated variation can be regarded as some simultaneous simple $\sin ()$ variation. Fig. 3 gives the $X(t)$ as $a=-0.5$ and $a=1$. Take $X(t)$ to Eq.(2),we have

$$
\operatorname{SERv}(t)= \begin{cases}S E R 0 & t \% T \leq T-T_{v} \\ S E R 0 e^{A \times \sin \left(\pi\left(t-T+T_{v}\right) / T_{v}\right)} & t \% T>T-T_{v}\end{cases}
$$

Here we make $A=C X^{*} a, C x$ is $S E R$ variation per unit $X(t)$ variation, $a$ is $X(t)$ total variation, so $A$ is $S E R$ total variation for $X(t)$. Take altitude as an example, $C X=0.8\left(S E R\right.$ increased with $e^{0.8}$ time per kilo-meter increased), suppose $a=5 \mathrm{KM}$, so $A=4$, meaning $S E R$ increased to $e^{4}$ time while $X(t)$ increased. Fig. 4 gives $\operatorname{SERv}(t)$ examples for $A=-1, A=0.5$ and $A=3$.

We can get number of errors occurred one cycle $T$ by cumulative $\operatorname{SERv}(t)$ from 0 to $T$, and we can see number of errors at $A=3$ is greater than $A=0$, and that at $A=0$ is also greater than $A=-1$. Overhead is greatly relative with errors number, and this paper is going to study overhead due to $S E R$ variability, so we use a factor $K$ to equalize errors of $\operatorname{SER} v(t)$ in Eq.(5).

$$
\operatorname{SERv}(t)= \begin{cases}S E R 0 \times K & t \% T \leq T-T_{v} \\ S E R 0 \times K e^{A \times \sin \left(\pi\left(t-T+T_{v}\right) / T_{v}\right)} & t \% T>T-T_{v}\end{cases}
$$

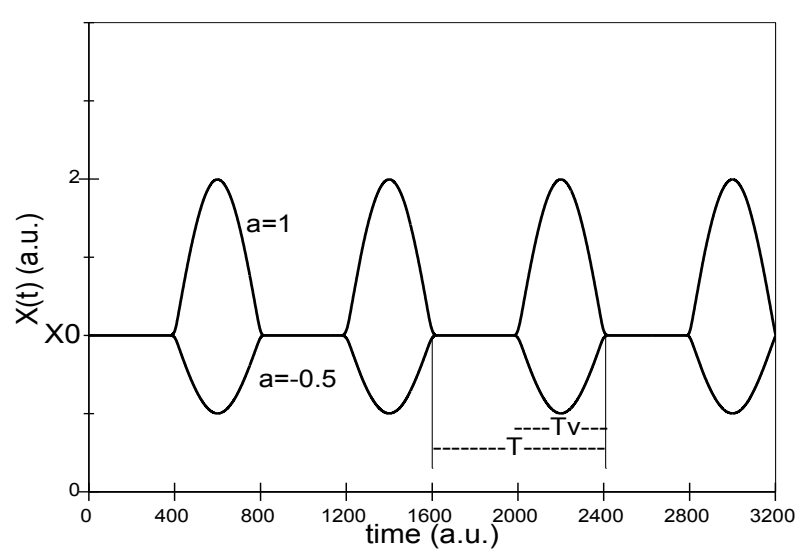

Fig. 3. Examples of $X(t)$ variation. 


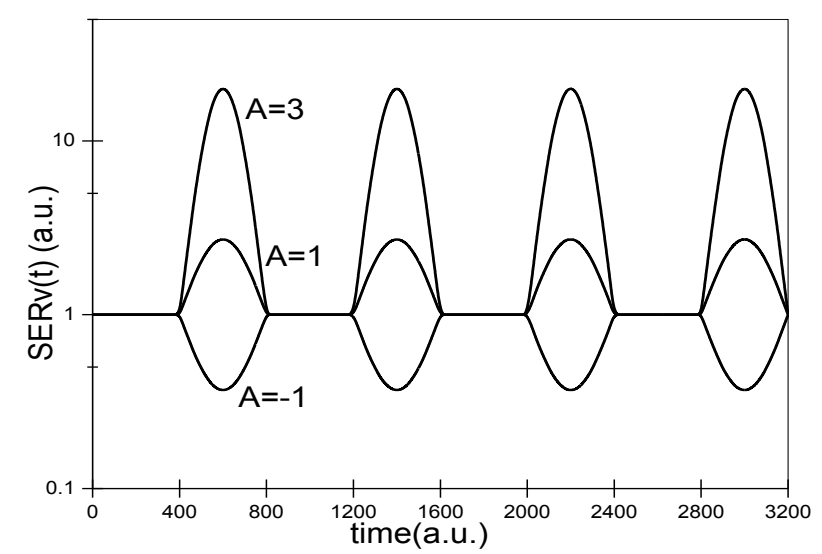

Fig. 4. $S E R(t)$ variation.

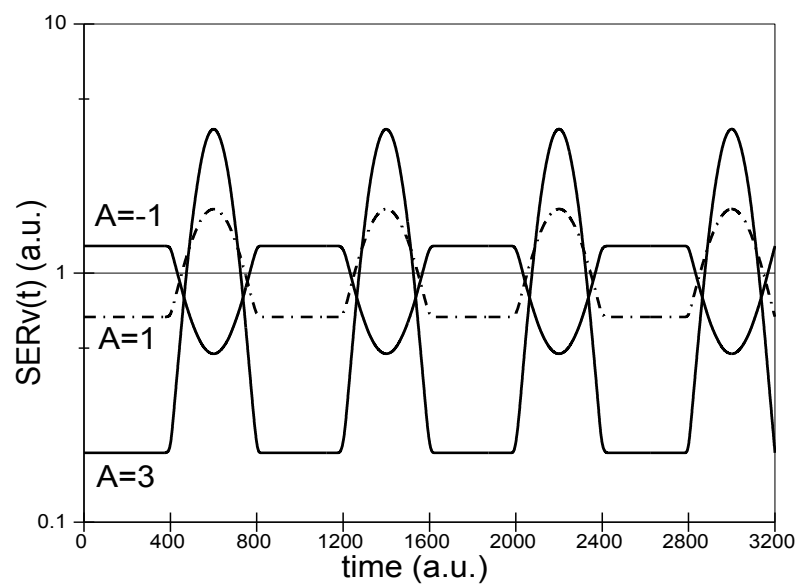

Fig. 5. $S E R(t)$ variation equalized.

Number of errors in every $T$ for constant $S E R$ is $\int_{0}^{T} S E R c(t) d t=S E R 0 \times T$. We equalize errors for $S E R v(t)$ as following function $\int_{0}^{T} S E R v(t) d t \times K=S E R 0 \times T$, so $K=S E R 0 \times T / \int_{0}^{T} S E R v(t) d t$ and value of $K$ is determined by $A$ and $T_{v} / T$. Fig. 5 shows the $\operatorname{SERv}(t)$ equalized.

$S E R$ variation we studied in this paper is based on Eq.(5), we summery the parameters as following:

- SER0: the average SER in every cycle $T$.

- A: amplitude of $S E R$ variation, defined as maximal SER/minimum $S E R=e^{|A|}$. We categorize $S E R$ variation as increased variation if $A>0$ and decreased variation if $A<0$.

- $T_{v}: T_{v}$ is duration for variation sustained.

- $p n: p n=T_{v} / T$ is time proportion of variation, $T$ is cycle time of variation.

Our goal is to study how the parameters (SER0, $A, T_{v}, p n$ ) impact checkpoint overhead for increased and decreased variation respectively. $S E R$ variation actually is more complicated than we modeled in Eq.(5), however, we can regard a complicated variation as some simultaneous simple variation.

\section{SER Prediction}

SER is usually calculated by number of errors occurred/time systems have run. Due to variation, we could not use errors occurred long time ago to predict real-time SER, just as Fig. 6(a) shown. Simulated results in Fig. 6(a) manifest delay between theoretical SER and predicted SER using errors occurred in 200 hours. So the key to $S E R$ prediction is proper time of errors occurred, and we define periods employed to predict $S E R$ as prediction window (PW). 

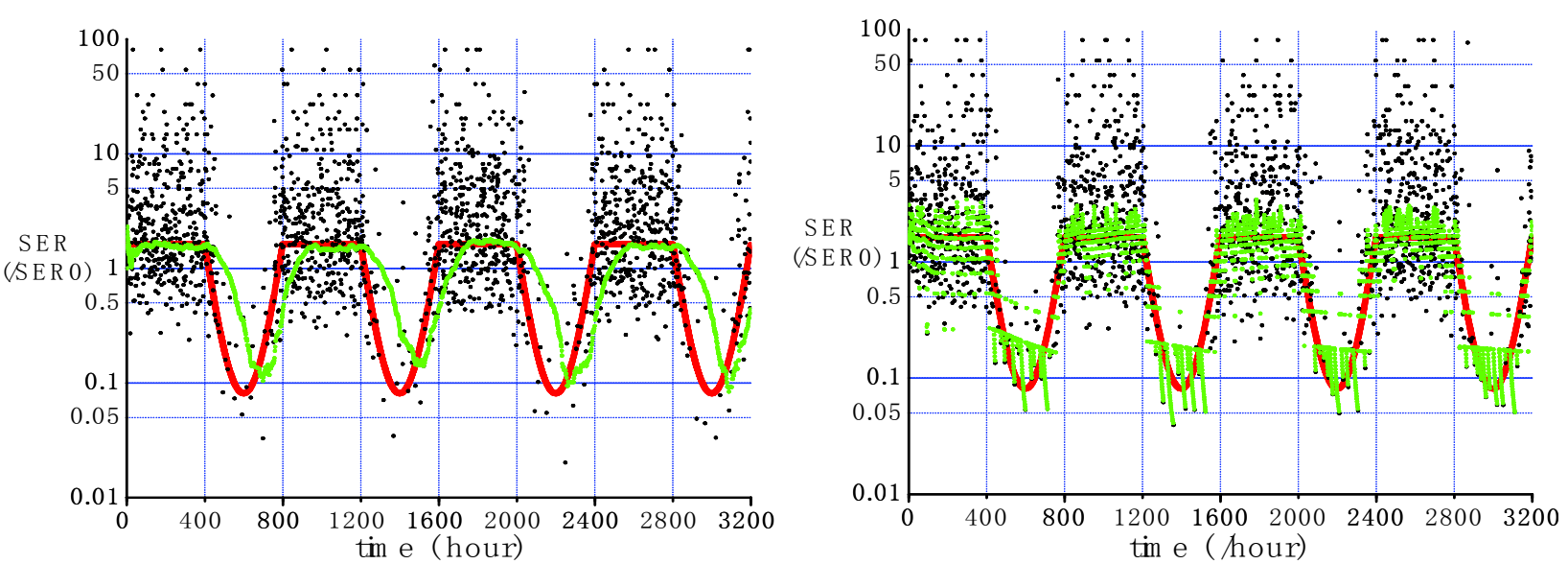

Fig. 6. SER prediction; (a) Prediction window $=200 \mathrm{~h}$, deviation cause by delay; (b) Prediction window $=10 \mathrm{~h}$, deviation cause by random. (Red line is theoretical SER as Eq.(5), black points are simulated errors occurrence based red line, green points are $S E R$ we predicted based black points. Suppose $S E R 0=1 / 100 \mathrm{~min}^{-1}$ ).

Tow reason decreasing accuracy of SER prediction is random of errors occurred and delay due to SER variation. Errors occurred randomly (as Fig. 6(b)) and we need many errors to amortize deviation of SER. Whereas we should use errors occurred as current as possible for less delay deviation. Unfortunately, the two ways to decrease deviation is usually contrary. More errors to amortize random deviation indicate longer time ago errors to be used, which will obviously worsen prediction accuracy under low SER.

To balance the deviation cause by delay and random, we employ shorter PW when SER is largely changing and longer PW when SER relative constant, as shown in Table 2.

Table 2. Algorithm of Dynamic Prediction Window (DPW)

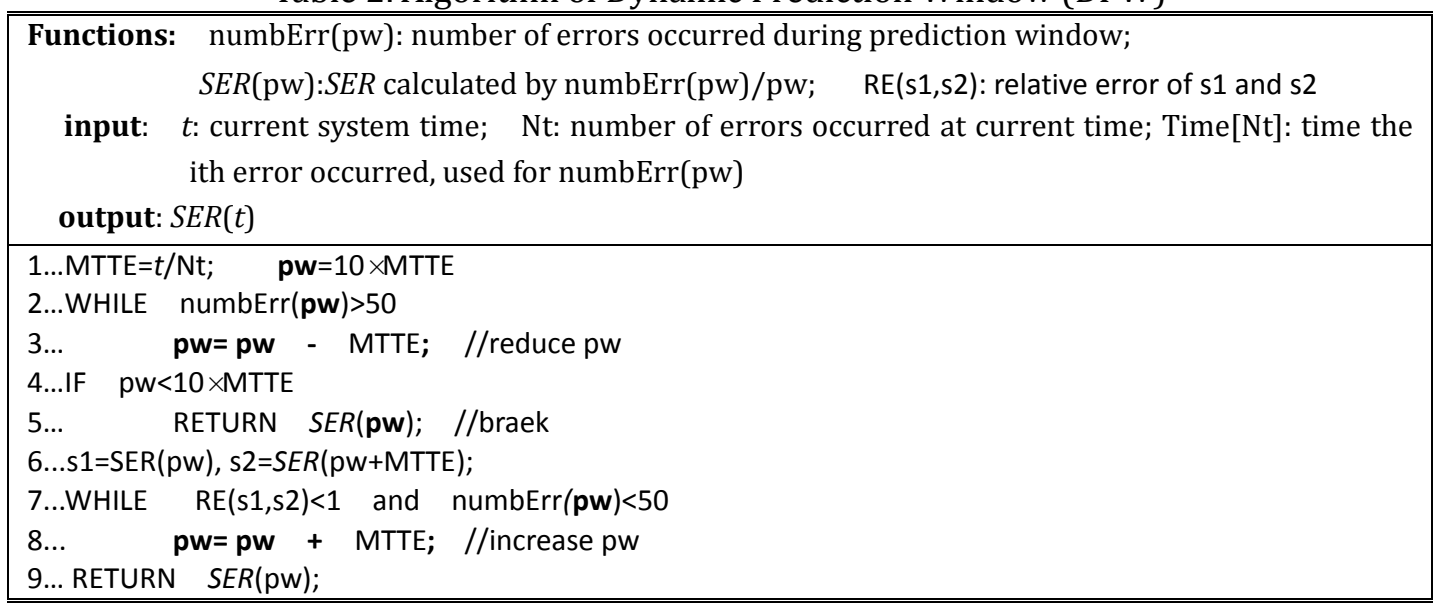

DPW is dynamically increase or decrease the prediction window based $\operatorname{RE}(\mathrm{s} 1, \mathrm{~s} 2)$ and numbErr $(p w)$, making it works well for both continuous and variation. DPW is low-overhead and need no more system information, except the time of each error occurred.

We use error ratio between predicted SER and theoretical SER (shown as Eq.(5)) to measure precision of prediction, Error ratio of at $t$ is defined as

$$
\operatorname{ER}(t)=\frac{\operatorname{MAX}\left(S E R_{-} p r e(t), S E R_{-} \mathrm{OPT}(t)\right)}{\operatorname{MIN}\left(S E R \_p r e(\mathrm{t}), S E R_{-} \mathrm{OPT}(t)\right)}
$$

We refer error ratio to overestimating if $S E R_{-} \operatorname{pre}(t)>S E R_{-}$OPT $(t)$ or underestimating if 
$S E R \_$pre $(t)<S E R \_O P T(t)$. Sum of ER as entire application ran is

$$
\mathrm{ER}=\sum_{i=1}^{N}\left(\operatorname{ER}\left(t_{i}\right) \times \frac{T \mathrm{ex}_{i}}{T \text { solve }}\right)
$$

Here $N$ is number of checkpoint intervals, $t_{i}$ is the time $i$ th interval started, $T_{\text {ex }}(i)$ is length of $i$ th interval, $T_{\text {solve }}$ is sum of $T_{\text {ex. }}$ Sum of ER is great than 1 , the larger means the worse precision of prediction.

\section{Simulation Methods}

We simulate the checkpoint mechanism as following steps in Table 3. The simulation generates pseudo-random errors in a Poisson distribution.

Based on [6], [14], basic parameters for checkpoint are given: $S E R 0=1 / 100 \mathrm{~min}^{-1}, T_{\text {total }}=3200 \mathrm{~h}$, time overhead to update checkpoint is $2 \mathrm{~min}$, time to recovery errors is $4 \mathrm{~min}$. $T$ and $T_{v}$ are relative to $S E R 0$ (enough errors should occur to show SER variation in every $T$ ), here we suppose $T=800 \mathrm{~h}, T_{v}=400 \mathrm{~h}$.

Table 3. Method to Simulate Checkpoint

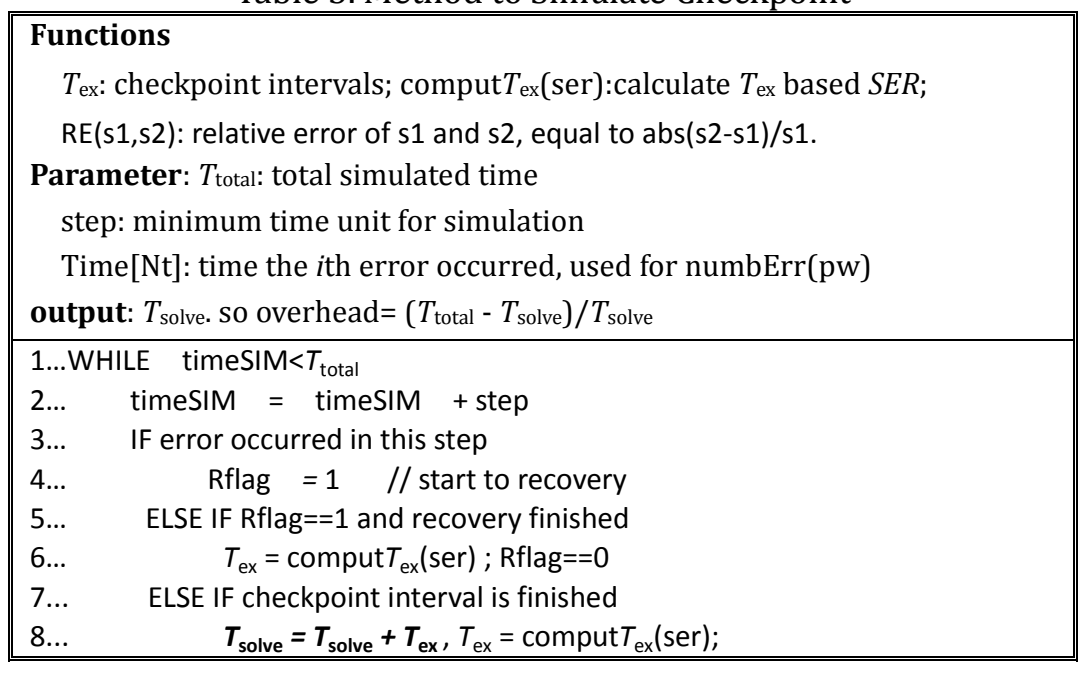

\subsection{Determining Simulation Parameters}

Parameters we need to determine is: how less should simulation step be (which will determine the simulation precision) and how many times should the simulation need to repeat for each case (which will average the simulation deviation).

The step of simulation has great influence on result precision. For Poisson distribution simulated, only one error occurred per simulation step at most, so relative error of number occurrence is $(\lambda \times s t e p$

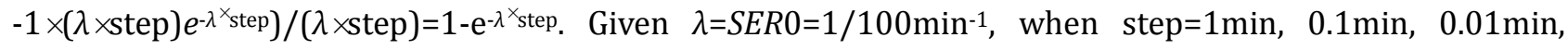
relative error is respectively $0.99 \%, 0.10 \%, 0.01 \%$, so we donate step $=0.1 \mathrm{~min}$ to expect relative error of number occurrence less than $0.1 \%$.

How many times we should simulated per case to average simulation result? According to [15], we denote $99 \%$ confidence level, $0.1 \%$ relative error, and get times $>3458$. We simulated 3500 times per case, so we can assure a $0.1 \%$ precision for errors simulated.

\subsection{Analysing and Optimizing Simulation Error}

The values we concerned with are number of errors really occurred and overhead of checkpoint, and we analyzing simulation error by comparing the true value with outcome of simulation, which is listing in Table 4. From basic parameters for checkpoint, true value of errors number is $3200 \times 60 / 100=1920$. We calculate true value of overhead by model given by Daly [6]. 
Table 4. Errors Simulated Precision for Different Amplitude $A$

\begin{tabular}{c|c|c|c|c|c|c|c|c}
\hline \multirow{2}{*}{ method } & \multicolumn{4}{|c|}{ Number of errors } & \multicolumn{3}{c}{ Overhead (\%) and relative error(\%) } \\
\cline { 2 - 9 } & $A=0$ & $A=1$ & $A=3$ & $A=5$ & $A=0$ & $A=1$ & $A=3$ & $A=5$ \\
\hline true value & 1920 & 1920 & 1920 & 1920 & 28.00 & 27.76 & 27.06 & 26.89 \\
\hline simulated step=0.1min & 1918.73 & 1917.74 & 1911.71 & 1906.56 & 28.17 & 27.62 & 26.58 & 26.39 \\
& & & & & $(0.61)$ & $(0.50)$ & $(1.77)$ & $(1.85)$ \\
\hline $\begin{array}{c}\text { simulated step=MIN }\{0.1, \\
0.1 \times S E R 0 / S E R v(t)\}\end{array}$ & 1918.21 & \multirow{2}{*}{1918.97} & 1918.10 & 1918.54 & $\begin{array}{c}28.17 \\
(0.61)\end{array}$ & $\begin{array}{c}27.82 \\
(0.22)\end{array}$ & $\begin{array}{c}27.24 \\
(0.67)\end{array}$ & $\begin{array}{c}27.06 \\
(0.63)\end{array}$ \\
\hline
\end{tabular}

Table 4 shows that for $A=0$ simulation with $0.1 \mathrm{~min}$ step fits well for both errors number (relative error is $0.07 \%$ ) and overhead (relative error is $0.61 \%$ ). However, SER variation will affect the precision. Error number not good for $A=5$ (relative error $0.7 \%$ ), in which errors may occur much more in short time. We used a viable step for high $S E R$ to optimizing simulation error, as step is minimum of 0.1 and $0.1 \times \operatorname{SER} 0 / \operatorname{SERv}(t)$. The viable step fit very well for all value of $A$, making relative error of errors number less than $0.1 \%$ and overhead less than $1.0 \%$.

We simulated respectively for the following methods:

- FIX, fixed interval of checkpoint, the intervals are computed with the SERO and never change during system execution.

- SACP_OPT, SER adaptive intervals with the theoretical SER as Eq.(5), we use SACP_OPT to evaluate the optimal benefit of SACP.

- SACP_DPW, SER adaptive interval with SER predicted by DPW, in order to show the benefit can be achieved by SACP.

\section{Results}

In order to clearly describe overhead, we make the agreement with absolute improvement to be "overhead of FIX — overhead of SACP", relative improvement to be "(overhead of FIX — overhead of SACP)/overhead of FIX" and improvement to be "relative improvement" if not specially speaking.

\subsection{Average SER}

Fig. 7 shows how overhead vary with SER0 from $1 / 800 \mathrm{~min}^{-1}$ to $1 / 12.5 \mathrm{~min}^{-1}$. Results in Fig. 7 (1) show that overhead benefit between SACP_OPT and FIX quickly increase from $1.3 \%$ to $19 \%$. Overhead improvement of SACP_OPT increase from $15 \%\left(\right.$ SER $\left.0=1 / 100 \mathrm{~min}^{-1}\right)$ to $17 \%\left(S E R 0=1 / 12.5 \mathrm{~min}^{-1}\right)$. Results also show improvement nearly constant with SER less than $1 / 100 \mathrm{~min}^{-1}$, that is to say SACP_OPT can always improve overhead by $15 \%$ even for very low $S E R$.

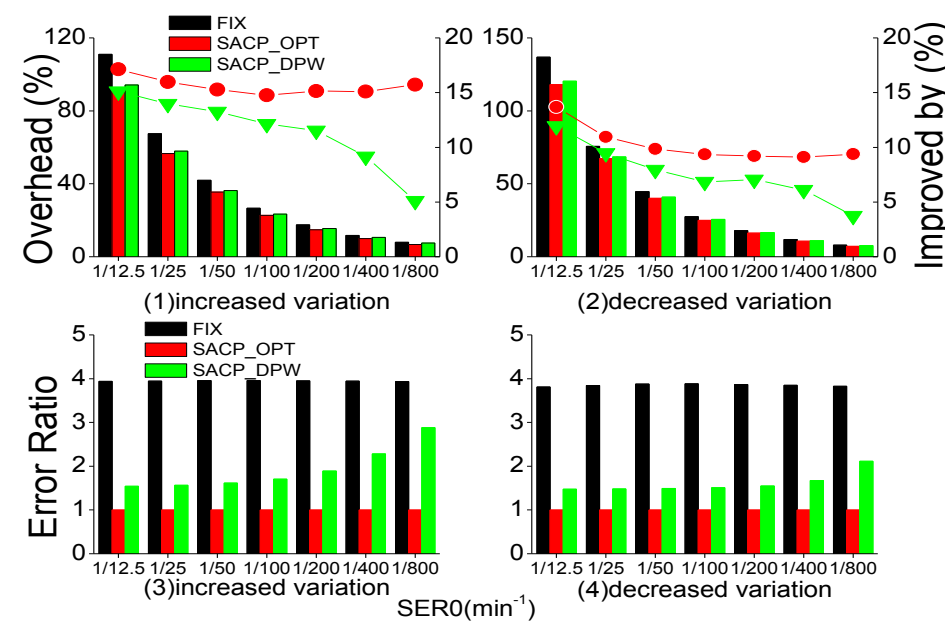

Fig. 7. Overhead and improvement for $S E R 0$ with $|A|=3$. 
Overhead improvement of SACP_DPW largely increases form $5 \%$ to $15 \%$ as SER0 higher. SACP_DPW hardly improves overhead with SER less than $1 / 800 \mathrm{~min}^{-1}$.because DPW predicts real-time SER based on errors, lower $S E R$, less errors occurred at same periods, less precision for SER prediction.

We recall error ratio(ER) defined in Eq. (7) that ER of SACP_OPT is always 1 and more ER means more relative error of SER compared with SACP_OPT. Error ratio in Fig. 7(3) clearly shows that ER of SACP_DPW stays less than 2 with $S E R 0<200 \mathrm{~min}^{-1}$ and increases to 2.8 in $1 / 800 \mathrm{~min}^{-1}$ and probably is greater than FIX with $S E R 0>3200 \mathrm{~min}^{-1}$ under which condition that so few errors occurred for SER prediction.

Fig. 7(2) and (4) show the same thing, summarily increased variations get better overhead improvement than decrease but worse error ratio of SER prediction.

\subsection{Variation Amplitude $A$}

Results in Fig. 8 show that as amplitude getting larger, overhead of SACP_OPT and SACP_DPW obviously decrease. When $A$ less than 1, SACP_DPW is worse than FIX, while $A$ larger than 2 SACP_DPW improve FIX overhead as much as by $25 \%$. Results of error radio show that DPW works well, especially for decreased variation with ER less than 2 for all $A$.

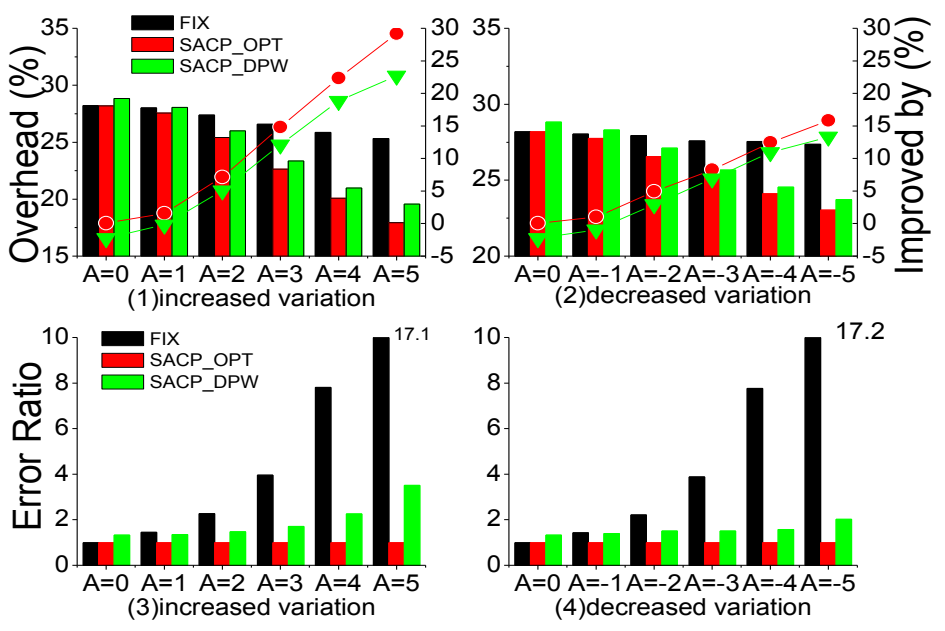

Fig. 8. Overhead and improvement for $A$ with $S E R 0=1 / 100 \mathrm{~min}^{-1}$.

Overhead and corresponding improvement: overhead (bar shown with left axis) and corresponding improvement (line shown with right axis) for (1)overhead and corresponding improvement for increased variation (2)overhead and corresponding improvement for decreased variation (3)error ratio for increased variation (4)error ratio for decreased variation., with $|A|=3, S E R 0=1 / 100 \mathrm{~min}^{-1}$.

\subsection{Variation Duration $T_{v}$}

Results in Fig. 9 show nearly constant improvement of SACP_OPT, while improvement of SACP_DPW obviously decrease as $T_{v}<200 \mathrm{~h}$. If $T_{v}$ is less than $25 \mathrm{~h}$, SACP_DPW will get no improvement. So few errors occurred in such a short periods make the variation maybe finished before SACP_DPW senses the variation.

\subsection{Variation Proportion pn}

Results in Fig. 10 show benefits increase as $p n$ increase to 4/8, however, a more complicated trend after that for increased variation and decreased variation. The reasons are shown in Fig. 11.

Error ratio and overestimating error ratio shown in black and red bar are increase for both variations, however, proportion of overestimating error ratio decreases from $100 \%$ to $92 \%$ for increased variation but increases from $0 \%$ to $95 \%$ for decreased variation. Just as many research mentioned overestimating checkpoint interval will result in more overhead than underestimating, that is why overhead of increased 
variation and decreased variation show differently trend in Fig. 10.
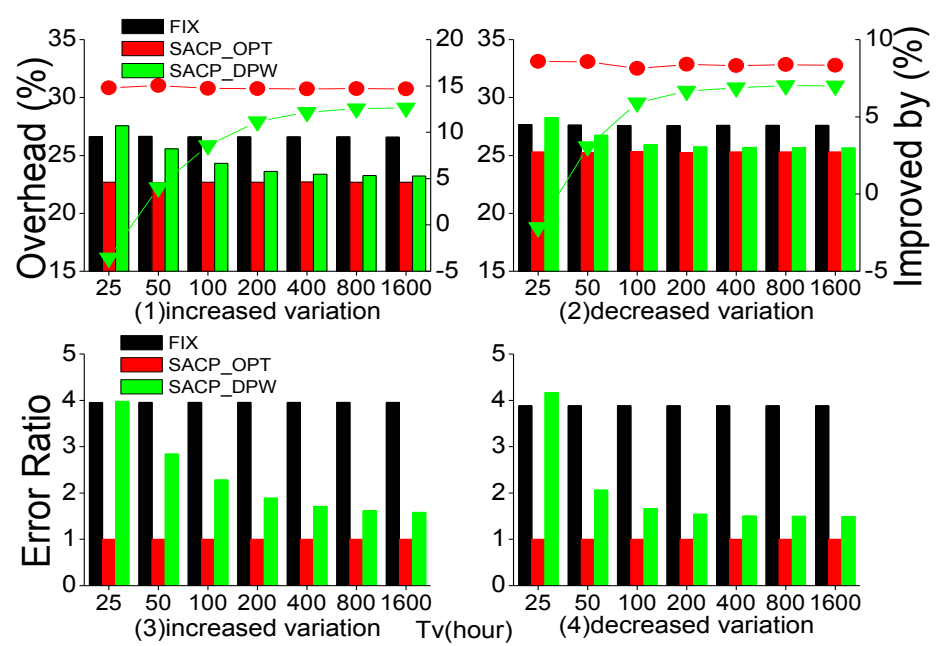

Fig. 9. Overhead and improvement for $T_{v}$ with $p n=1 / 2$.
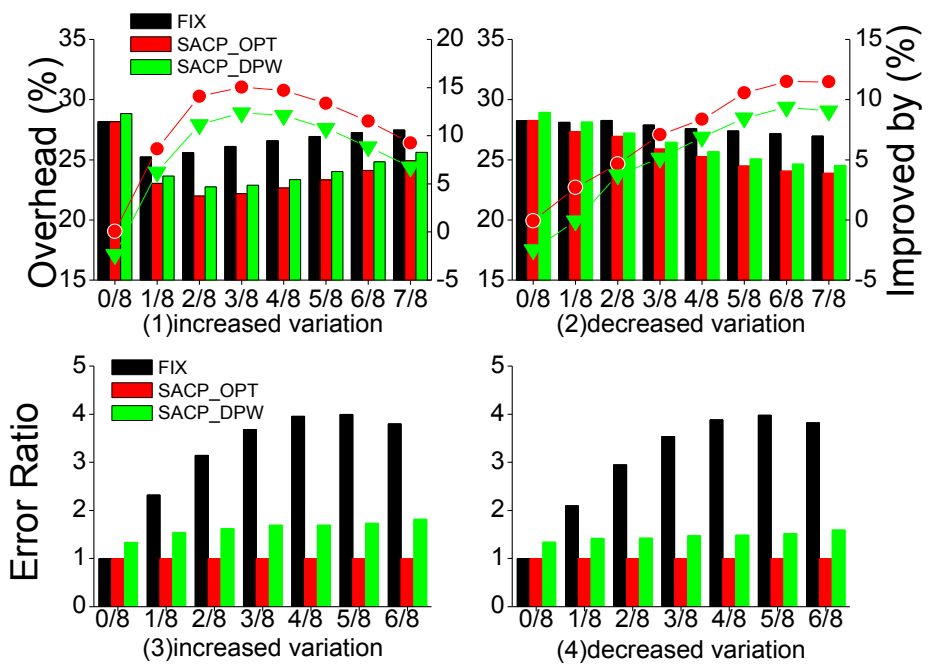

Fig. 10. Overhead and improvement for $p n$ with $T_{v}=400 \mathrm{~h}$.

Overhead and corresponding improvement: overhead (bar shown with left axis) and corresponding improvement (line shown with right axis) for (1)overhead and corresponding improvement for increased variation (2)overhead and corresponding improvement for decreased variation (3)error ratio for increased variation (4)error ratio for decreased variation., $T_{v}=400 \mathrm{~h}, p n=1 / 2$.

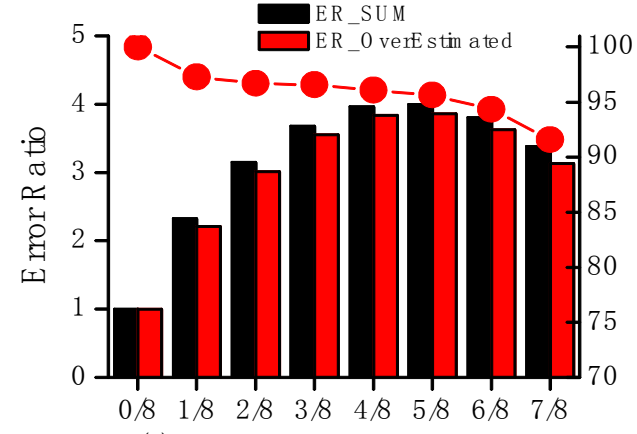

(a) increased continuous variation

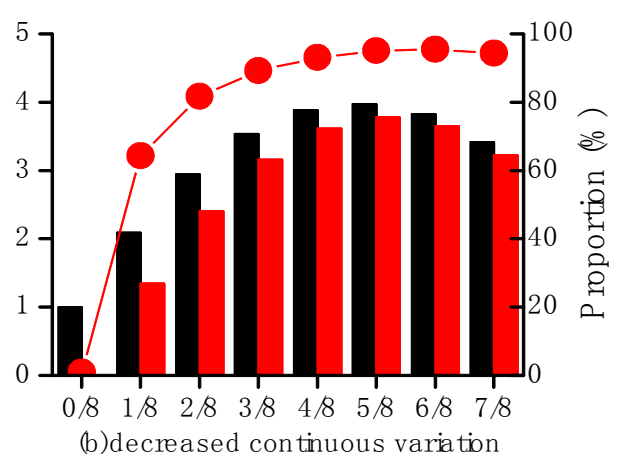

(b)decreased continuous variation

Fig. 11. Error ratio of FIX for $p n$. 
Error ratio (bar shown with left axis, sum of overestimating and underestimating error ratio labeled as ER_SUM and overestimating error ratio labelled as ER_OV) and overestimating proportion (line shown with right axis) for (a) increased variation (b) decreased variation\}.

\section{Conclusion}

Soft errors are increasingly important threats to the reliability of integrated circuits. Chips manufactured in advanced technologies show variation in SER caused by variation in the process parameters. Ongoing reduction of feature sizes and complexity of operating environment, SER variation is increasingly manifesting. Checkpoint is the most popular recovery method, and the intervals of checkpoint can obviously influence performance. But optimal intervals of checkpoint are determined on SER. SER adaptive checkpoint which dynamically match checkpoint intervals with real time $S E R$ can improve checkpoint overhead under variable SER. But benefit of SACP is relative with SER variation, so in this paper we evaluate impact of SER variability on $S E R$ adaptive checkpoint.

This paper is focusing on SER variation which has rarely been studied. Based on how SER affected by temperature, voltage, radiation pressure and so on, we model $S E R$ variation with four parameters : average SER SER0, variation amplitude $A$, variation duration $T_{v}$ and variation proportion $p n$. With an exact simulation, we come out how much each parameter impacts the performance overhead of present checkpoint and SER adaptive checkpoint. We proposal a way to predict SER based dynamic prediction window (DPW), showing practical benefits of SACP. We categorize SER variation to increased variation and decreased variation. We give how much the parameters (SER0, $A, T_{v}, p n$ ) impact checkpoint overhead for those four variability respectively.

Actual SER of systems may be more complicated than we modeled in this paper, however, we can regard a complicated variation as some simple variation acted simultaneously. Results in this paper can be referred to systems on weather SER variation need be considered or how much benefit SER adaptive checkpoint maybe achieved.

\section{Acknowledgment}

The authors gratefully acknowledge supports from National Nature Science Foundation of China under NSFC No. 61402504, 61033008, 61272145, and 61103080; National High Technology Research and Development Program of China (863 Program) under No. 2012AA012706; Research Fund for the Doctoral Program of Higher Education of China under SRFDP No. 20124307130004.

\section{References}

[1] Borkar, S. (2005). Designing reliable systems from unreliable components: The challenges of transistor variability and degradation. IEEE Micro, 25(16), 10-16.

[2] Nicolaidis, M. (2011). Soft Errors in Modern Electronic Systems. New York, USA: Springer Science and Business Media.

[3] Heijmen, T. (2007). Spread in alpha-particle-induced soft-error rate of 90-nm embedded SRAMs. Proceedings of the 13th IEEE International on-Line Testing Symposium (pp. 131-136). Heraklion, Crete, Greece.

[4] Heijmen T., Roche P., Gasiot, G., Forbes, K. R., \& Giot, D. (2007). A comprehensive study on the soft-error rate of flip-flops from 90-nm production libraries. IEEE Transactions on Device and Materials Reliability, $7(1), 84-96$.

[5] Zielger, J. F., \& Puchner, H. (2004). SER-history, trends, and challenges: A guide for designing with memory ICs. Cypress Semiconductor Corporation. 
[6] Daly, J. T. (2006). A higher order estimate of the optimum checkpoint interval for restart dumps. Future Generation Computer Systems, 22, 303-312.

[7] Hazucha, P., \& Svensson, C. (2000). Impact of CMOS technology scaling on the atmospheric neutron soft error rate. IEEE Transactions on Nuclear Science, 47(6), 2586-2594.

[8] JEDEC Solid State Technology Association. (2006). JEDEC standards on measurement and reporting of alpha particle and terrestrial cosmic ray induced soft errors in semiconductor devices.

[9] Chandra, V, \& Aitken, R. (2008). Impact of technology and voltage scaling on the soft error susceptibility in nanoscale CMOS. Proceedings of IEEE International Symposium on Defect and Fault Tolerance of VLSI Systems (pp. 114-122). Boston, MA, USA.

[10] Jagannathan, S., Diggins, Z., Mahatme, N. N., Loveless, T. D., Bhuva, B. L., et al. (2012). Temperature dependence of soft error rate in flip-flop designs. Proceedings of IEEE International Reliability Physics Symposium, Anaheim, CA.

[11] Zhang, C., Deng, S., \& Ning, H. (2012). A local checkpoint mechanism for on-board computing. Proceedings of International Conference on Information Science and Technology (pp. 520-526). Hubei, China.

[12] Xiong, L., \& Tan, Q. (2011). Data flow error recovery with check pointing and instruction-level fault tolerance. Proceedings of International Conference on Parallel and Distributed Computing, Applications and Technologies (pp. 79-85). Gwangju, Korea.

[13] Gerofi, B., \& Ishikawa, Y. (2011). Workload adaptive checkpoint scheduling of virtual machine replication. Proceedings of the 2011 IEEE 17th Pacific Rim International Symposium on Dependable Computing (pp. 204-213). Pasadena, CA, USA.

[14] Jones, W. M., Daly, J. T., \& Debardeleben, N. (2010). Impact of sub-optimal checkpoint intervals on application efficiency in computational clusters. Proceedings of the 19th ACM International Symposium on High Performance Distributed Computing (pp. 276-279). New York, NY, USA.

[15] Shubu, M. (2008). Architecture Design for Soft Errors. San Francisco, CA: Morgan Kaufmann Publishers Inc.

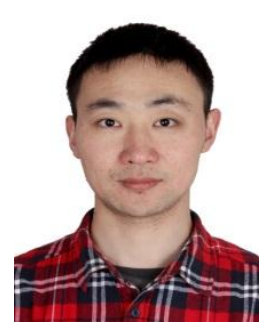

Wentao Jia was born in 1985, he is a Ph.D. candidate in the National Laboratory for Parallel and Distributed Processing, National University of Defense Technology, Changsha, China. His research interests include computer architecture and fault-tolerant computing.

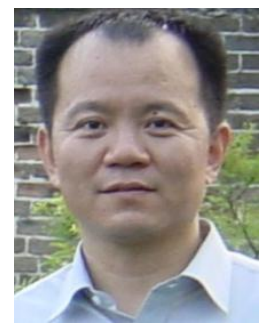

Chunyuan Zhang was born in 1964, he is a professor with the National Laboratory for Parallel and Distributed Processing, National University of Defense Technology, Changsha, China. His research interests include computer architecture, parallel programming, low power design, embedded systems, media processing and scientific computing. 